\begin{tabular}{|c|l|}
\hline Title & A pplication of self-deconvolution method to shift-and-add solar imaging \\
\hline Author(s) & Sudo, Y.; Baba, N.; Miura, N.; Ueno, S.; Kitai, R. \\
\hline Citation & APPLIED OPTICS, 45(12), 2707-2710 \\
\hline Issue Date & 2006-04 20 \\
\hline Doc URL & http://hdl.handle.net/2115/14478 \\
\hline Rights & (c) 2006 O ptical Society of A merica. \\
\hline Type & article (author version) \\
\hline File Information & AO_05.pdf \\
\hline
\end{tabular}

Instructions for use 


\section{Application of self-deconvolution method to shift-and-add solar imaging}

Yoshifumi Sudo $^{1)}$, Naoshi Baba ${ }^{1)}$, Noriaki Miura ${ }^{2)}$, Satoru Ueno ${ }^{3)}$, and Reizaburo Kitai ${ }^{3)}$

1) Department of Applied Physics, Graduate School of Engineering, Hokkaido University, Sapporo 060-8628, Japan. Y. Sudo is now with Opt-Electronics Module Development Department, Ricoh Co., Ltd., Shimo-imaizumi 810, Ebina 143-8555, Japan.

2) Department of Computer Sciences, Kitami Institute of Technology, Kitami 090-8507, Japan

3) Hida Observatory, Graduate School of Science, Kyoto University, Takayama 506-1314, Japan

A shift-and-add (SAA) operation is conducted to reconstruct a high-spatial-resolution image from atmospherically degraded solar images. The self-deconvolving data reconstruction algorithm (SeDDaRA) is used to augment high-spatial-frequency components in solar speckle images and rectify background component resulted from the SAA operation. Self-deconvolved solar speckle images are shift-and-added and the resulted image shows high-spatial-resolution features. 


\section{c 2005 Optical Society of America}

OCIS codes: 030.6610 (Stellar speckle interferometry), 100.1830 (Deconvolution), 100.3020

(Image reconstruction-restoration), 100.3010 (Image reconstruction techniques), 100.2000

(Digital image processing), 030.7060 (Turbulence).

\section{Introduction}

Terrestrial atmospheric turbulence hampers high-spatial-resolution imaging of the sun from the ground. Adaptive optics (AO) ${ }^{1,2}$ can mitigate the atmospheric turbulence in real time. On the other hand there are various a posteriori methods to reconstruct high-spatial-resolution image from atmospherically degraded solar images. Stellar speckle imaging methods have been applied to solar observations. ${ }^{3,4}$ Phase diversity imaging method has been shown useful for solar observations. ${ }^{5-7}$ Blind deconvolution technique has been also applied to solar speckle images. ${ }^{8,9}$ Among these methods a shift-and-add (SAA) method $^{10,11}$ in speckle solar imaging can be regarded as the simplest one.

The key operation in SAA is to shift each data frame to center on the peak position of the instantaneous point-spread function (PSF). However, it is difficult to find the peak position of the PSF from an image of an extended object such as the sun. Therefore, some alternative method is required to find the peak of the PSF. Cross-correlation of instantaneous images with the reference image, which might be the best-shot image over the observed ones, is usually conducted to determine the shifting vector for the SAA operation. Baba et al. ${ }^{12}$ used one solar granulation or a part of that, namely a small part of the object, for the cross-correlation and produced an SAA solar image. The determination of the shifting vector, however, depends on the 
selection of the reference solar granulation and, therefore, the quality of the SAA reconstruction may vary. It is desirable that the shifting vector for the SAA operation is determined free from the selection of a reference pattern.

The other problem in the SAA reconstruction is debiasing the background component, since an SAA image is formed by adding many data frames and the spread of the speckle pattern of the instantaneous PSF causes accumulation of the background component. In the former paper subtraction of the background component was conducted by using a centroid-tracking SAA image. ${ }^{13}$ However, two SAA images, namely correlation-peak tracking and centroid tracking, are necessary for image reconstruction in this method.

Recently Sudo and Baba ${ }^{14,15}$ have proposed an SAA imaging method by use of the selfdeconvolving data reconstruction algorithm (SeDDaRA). ${ }^{16,17}$ In this method each data frame is self-deconvolved and the shifting vector for the SAA operation is determined by crosscorrelating each data frame with a self-deconvolved reference frame. The SAA of selfdeconvolved data frames leads to debiased reconstruction. In this paper we report an SAA reconstruction of solar images by use of SeDDaRA.

\section{Method}

The $i$-th speckle image $g_{i}(x, y)$ can be written as

$$
g_{i}(x, y)=f(x, y) * h_{i}(x, y)+n_{i}(x, y)
$$

where $f(x, y), h_{i}(x, y)$, and $n_{i}(x, y)$ represent an object, an instantaneous PSF, and an additive noise term, respectively, and * denotes two-dimensional convolution. It should be noted that Eq. (1) holds only in the isoplanatic region where the PSF can be regarded invariant. In case of solar 
image processing it is necessary to partition the image into isoplanatic regions. The Fourier transform of Eq. (1) is

$$
G_{i}(u, v)=F(u, v) H_{i}(u, v)+N_{i}(u, v) .
$$

The deconvolution can be done by using a pseudo Wiener filter $D_{i}(u, v)$,

$$
D_{i}(u, v)=\frac{H_{s i}^{*}(u, v)}{\left|H_{s i}(u, v)\right|^{2}+\varepsilon_{i}},
$$

where $\varepsilon_{i}$ is a constsant. Following SeDDaRA $H_{s i}(u, v)$ is estimated as

$$
H_{s i}(u, v)=K_{d i} \operatorname{Sm}\left\{G_{i}(u, v)-\left.N_{i}(u, v)\right|^{\alpha}\right\}
$$

where $K_{d i}$ is a positive constant to ensure $\left|H_{s i}(u, v)\right| \leq 1, \operatorname{Sm}\{\}$ denotes a smoothing operation, and $\alpha$ an estimating parameter $(0 \leq \alpha \leq 1)$. The parameter $\alpha$ depends on the spatial frequency as noted in Ref. 16. However, we here set $\alpha(u, v)$ to 0.5 , because the estimation of $\alpha(u, v)$ needs the prior knowledge of the object itself and setting of $\alpha \approx 0.5$ usually results in good reconstruction as noted in Ref. 16 and also from our computer simulations. A low-pass Hanning window function is used for smoothing operation in our calculations. The noise spectrum is set to zero for the derivation of $H_{s i}(u, v)$ in Eq. (4), because the estimation of frequency-dependent noise term is difficult. We denote the self-deconvolved image as $\tilde{g}_{i}(x, y)$. Self-deconvolution leads to enhance high-spatial frequency components in an image.

A reference image is needed to determine the shifting vector in the SAA operation. The best-shot image is preferable as the reference image. The image with the least mean-square-error should be the best one but the calculation of the mean-square-error needs the intensity 
distribution of the object. Then, the best-shot image is selected by evaluating a root-meansquare-contrast (RMSC),

$$
R M S C=\sqrt{\sum_{x} \sum_{y} g_{i}{ }^{2}(x, y)-\left(\sum_{x} \sum_{y} g_{i}(x, y)\right)^{2}} / \sum_{x} \sum_{y} g_{i}(x, y) .
$$

The image with the highest RMSC value over data frames is taken as the reference image $r(x, y)$. The reference image is also self-deconvolved by using Eq. (3) to enhance high-spatial-frequency components and denoted as $\tilde{r}(x, y)$.

As described in Ref. 14 cross-correlation between self-deconvolved images tends to give the peak location of PSF, the most important information in the SAA. Then, cross-correlation between a self-deconvolved data frame $\tilde{g}_{i}(x, y)$ and the self-deconvolved reference image $\tilde{r}(x, y)$ is taken and the peak location is found. From this peak location the shifting vector $\left(x_{s i}\right.$, $\left.y_{s i}\right)$ in the SAA operation is determined. The debiased image is reconstructed by SAA of selfdeconvolved data frames;

$$
\tilde{g}(x, y)=\frac{1}{M} \sum_{i=1}^{M} \tilde{g}_{i}\left(x-x_{s i}, y-y_{s i}\right),
$$

where $M$ is the number of data frames.

\section{Observation}

The observations were made with a $60 \mathrm{~cm}$ domeless solar telescope at the Hida Observatory on 10 September, 2003. The focal length of the telescope is $f=32.2 \mathrm{~m}$. A G-band filter ( $\lambda=430.6 \mathrm{~nm}$, FWHM=2.2nm) and 3/4 reducing optical setup were used for observations of solar granulations and sun spots. We used a Peltier cooled CCD camera with 10-bit digitization as a detector. The pixel size of the CCD camera is $9.9 \mu m \times 9.9 \mu m$ and the number of pixels is $656 \times 494$. 
Figure 1 shows one observed frame around sunspots. The field size is $56.4^{\prime \prime} \times 42.5^{\prime \prime}$ ( $0.086 "$ / pixel). The diffraction-limited resolution of the telescope is $\approx 0.18^{\prime \prime}$ at $\lambda=430 \mathrm{~nm}$. The exposure time was 36.3ms. The RMSC (Eq. (5)) ranges from 0.095 to 0.103 and the mean value over 100 data frames is 0.098 .

\section{Image Reconstruction}

Solar image reconstruction cannot be done at a time, because the isoplanatic region is limited in solar observations. Here we set the isoplanatic angle to $\sim 9 "$ that corresponds to 100 pixels in the data frame. The self-deconvolved SAA is conducted on each isoplanatic region. The SAA reconstructed images are synthesized to form a large field-of-view image. Overlapping regions are needed to smoothly combine adjacent images. We reconstructed $16 \times 9$ regions, each of which has overlapping regions with the adjacent ones, and formed an image with a field of view of $37.8^{\prime \prime} \times 25.8^{\prime \prime}$. Figure 2(a) shows the best-shot image (evaluated by Eq. (5)) with the same field of view as that of the following reconstructions. Figure 2(b) shows a synthesized image of SAA reconstructed ones. In the SAA reconstruction we used 100 data frames and set $\varepsilon_{i}$ in Eq. (3) to $10^{-3}$. The resulted image reveals high contrasted features of solar granulations and sunspots compared with Fig. 2(a). However, the quality of the reconstructed image is not so good.

In the SAA reconstruction the quality of the reference frame is very important, because the shifting vector is determined by cross-correlating each data frame with the reference frame. It is expected that use of a better reference frame will lead to better SAA reconstruction. Therefore, we used the SAA reconstructed image as shown in Fig. 2(b) as the reference frame instead of the best-shot image in Fig. 2(a). And then the SAA reconstructed image is used as the reference frame in the next reconstruction. We iterated this process five times in total and obtained the 
reconstructed image as shown in Fig. 2(c). The reconstructed image, however, reveals hardly improvement as seen by comparing Figs. 2(c) with (b).

The reason why the reconstructed image is not good as expected from computer simulations in Ref. 14 can be attributed to bad seeing in observations. Christou examined the effectiveness of SAA reconstruction versus seeing. ${ }^{18}$ When seeing is bad, SAA does not result in good reconstruction. It is expected that a fairy good self-deconvolved SAA reconstruction will be obtained by using data frames under favorable seeing condition. Recently AO is being introduced to solar observations with telescopes of which aperture size is larger than $1 \mathrm{~m}$ diameter, but perfect wave-front correction is very difficult especially in visible region. The SAA reconstruction based on self-deconvolution will be useful for partially AO-corrected solar images.

\section{Conclusion}

We showed that incorporation of the SeDDaRA technique into the SAA is useful for restoring atmospherically degraded solar image. The SAA can be realized by using a tip-tilt mirror, ${ }^{19}$ namely the simplest operation in adaptive optics. When the shifting vector will be rapidly determined through self-deconvolving procedure as described above, a real-time SAA will be feasible. It should be noted that a superresolution method ${ }^{20}$ is available for improving solar image further.

We thank S. Sakuma for his help during our observations. 


\section{References}

1. D. S. Acton and R. C. Smithson, "Solar imaging with a segmented adaptive mirror," Appl. Opt. 31, 3161-3169 (1992).

2. T. R. Rimmel and R. R. Radick, "Solar adaptive optics at the National Solar Observatory," Proc. SPIE 3353, 72-81 (1998).

3. R. V. Stachnik, P. Nisenson, D. C. Ehn, R. H. Hudgin, and V. E. Schirf, “Speckle image reconstruction of solar features,” Nature 266, 149-151 (1977).

4. O. von der Lühe, “Speckle imaging of solar small scale structure I. Methods,” Astron. Astrophys. 268, 374-390 (1993).

5. M. G. Löfdahl and G. B. Scharmer, "Wavefront sensing and image restoration from focused and defocused solar images,” Astron. Astrophys. Suppl. Ser. 107, 243-264 (1994).

6. N. Baba, H. Tomita, and N. Miura, "Phase diversity imaging through atmospheric turbulence,” Opt. Rev. 1, 308-310 (1994).

7. R. G. Paxman, J. H. Seldin, M. G. Löfdahl, G. B. Scharmer, and C. U. Keller, "Evaluation of phase-diverse techniques for solar-image restoration,” Astrophys. J. 466, 1087-1099 (1996).

8. N. Miura, K. Ohsawa, and N. Baba, "Parallel blind deconvolution applied to solar image," Opt. Rev. 1, 208-210 (1994).

9. N. Miura and N. Baba, “Segmentation-based multiframe blind deconvolution of solar images,” J. Opt. Soc. Am. A12, 1858-1866 (1995).

10. R. H. T. Bates and F. M. Cady, “Towards true imaging by wideband speckle interferometry,” Opt. Commun. 32, 365-369 (1980). 
11. E. K. Hege, "First order imaging methods: an introduction,” in Diffraction-Limited Imaging with Very Large Telescopes (D. M. Alloin \& J. -M. Mariotti, eds., Kluwer, Dordrecht, 1989), pp. 141-155.

12. N. Baba, N. Miura, T. Sakurai, K. Ichimoto, D. Soltau, and P. Brandt, "Shift-and-add reconstruction of solar granulation images,” Sol. Phys. 188, 41-46 (1999).

13. S. Kuwamura, N. Baba, N. Miura, M. Noguchi, Y. Norimoto, and S. Isobe, "Preliminary observational results of wideband speckle spectroscopy,” in Proc. ESO Conf. HighResolution Imaging by Interferometry II (J. M. Beckers \& F. Merkle, eds., ESO, Garching, 1992), pp. 461-469.

14. Y. Sudo and N. Baba, "Self-deconvolution for shift-and-add imaging," Opt. Lett. 30, 13091311 (2005)

15. Y. Sudo and N. Baba, “Self-deconvolution for shift-and-add imaging: erratum,” Opt. Lett. 30, $2381(2005)$

16. J. N. Caron, N. M. Namazi, R. L. Lucke, C. J. Rollins, and P. R. Lynn, Jr., “Blind data restoration with an extracted filter function,” Opt. Lett. 26, 1164-1166 (2001).

17. J. N. Caron, N. M. Namazi, and C. J. Rollins, "Noniterative blind data restoration by use of an extracted filter function,” Appl. Opt. 32, 6884-6889 (2002).

18. J. C. Christou, "Image quality, tip-tilt correction, and shift-and-add infrared imaging,” Publ. Astron. Soc. Pacific 103, 1040-1048 (1991).

19. K. Hozumi, N. Baba, and N. Miura, "Projection speckle spectroscopy for a real-time mode,” Opt. Lett. 21, 887-889 (1996).

20. N. Miura, N. Baba, T. Sakurai, K. Ichimoto, D. Soltau, and P. Brandt, "Resolution improvement of solar images,” Sol. Phys. 187, 347-356 (1999). 


\section{Figure Captions}

Fig. 1 One of data frames ( $656 \times 494$ pixels). The field of view is $56.4^{\prime \prime} \times 42.5^{\prime \prime}$.

Fig. 2 (a) The best shot image over 100 data frames. The field of view is $37.8^{\prime \prime} \times 25.8^{\prime \prime}$. (b) The SAA reconstructed image, a synthesized image from $16 \times 9$ subregions. (c) Iteratively reconstructed SAA image, where the SAA reconstructed image, instead of the best-shot image, is used as the reference frame in the next iterative step. 


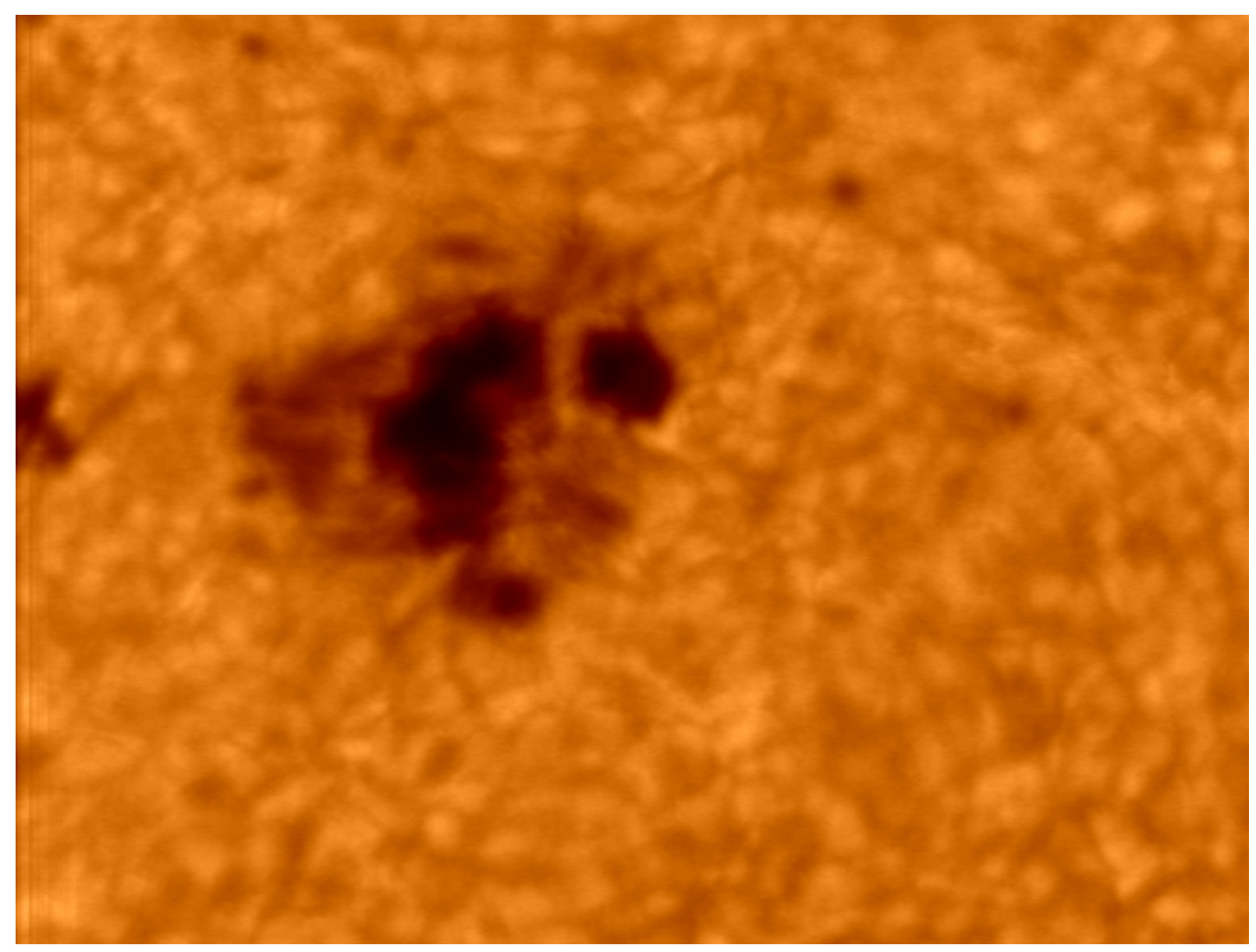

Fig. 1 One of data frames $\left(656 \times 494\right.$ pixels). The field of view is $56.4^{\prime \prime} \times 42.5^{\prime \prime}$. 


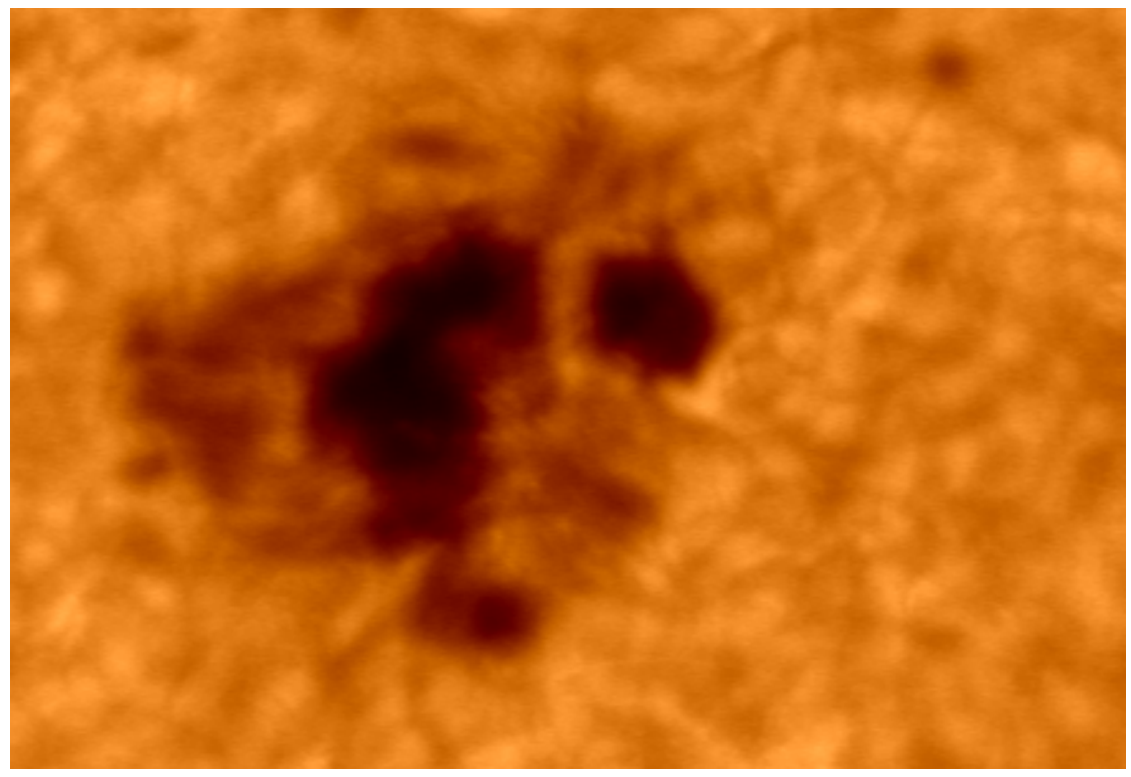

Fig. 2 (a) The best shot image over 100 data frames. The field of view is $37.8^{\prime \prime} \times 25.8^{\prime \prime}$. 


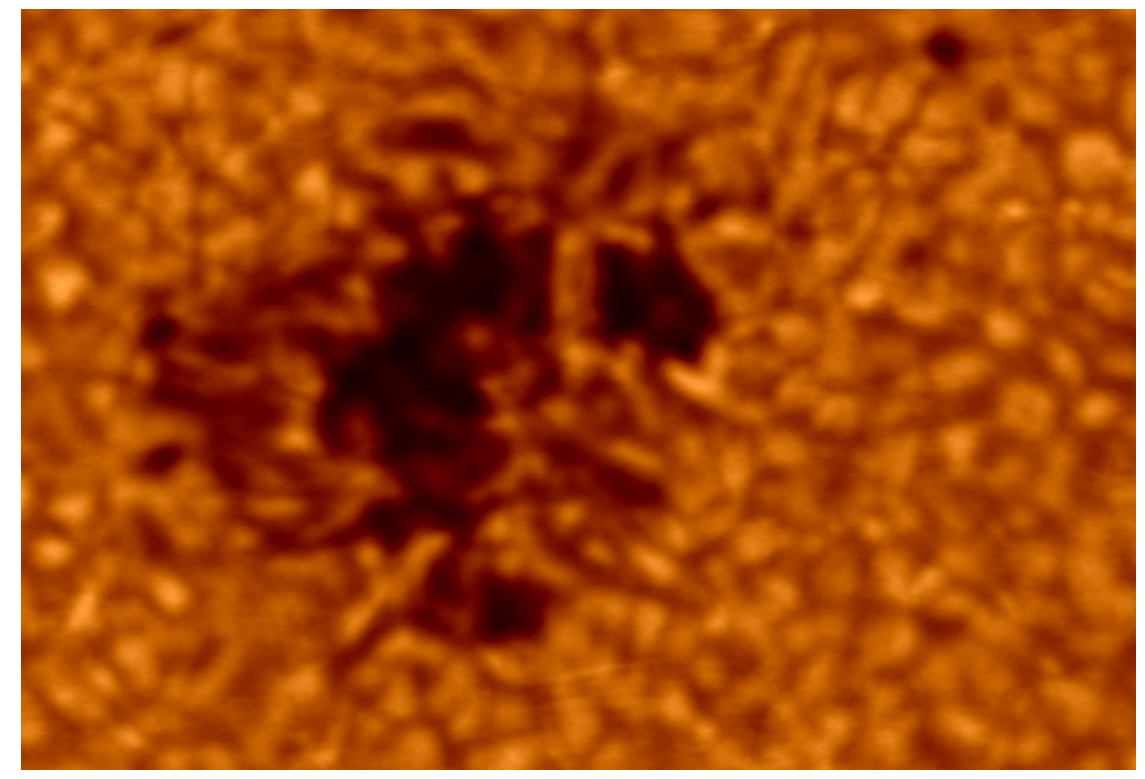

Fig. 2 (b) The SAA reconstructed image, a synthesized image from $16 \times 9$ subregions. 


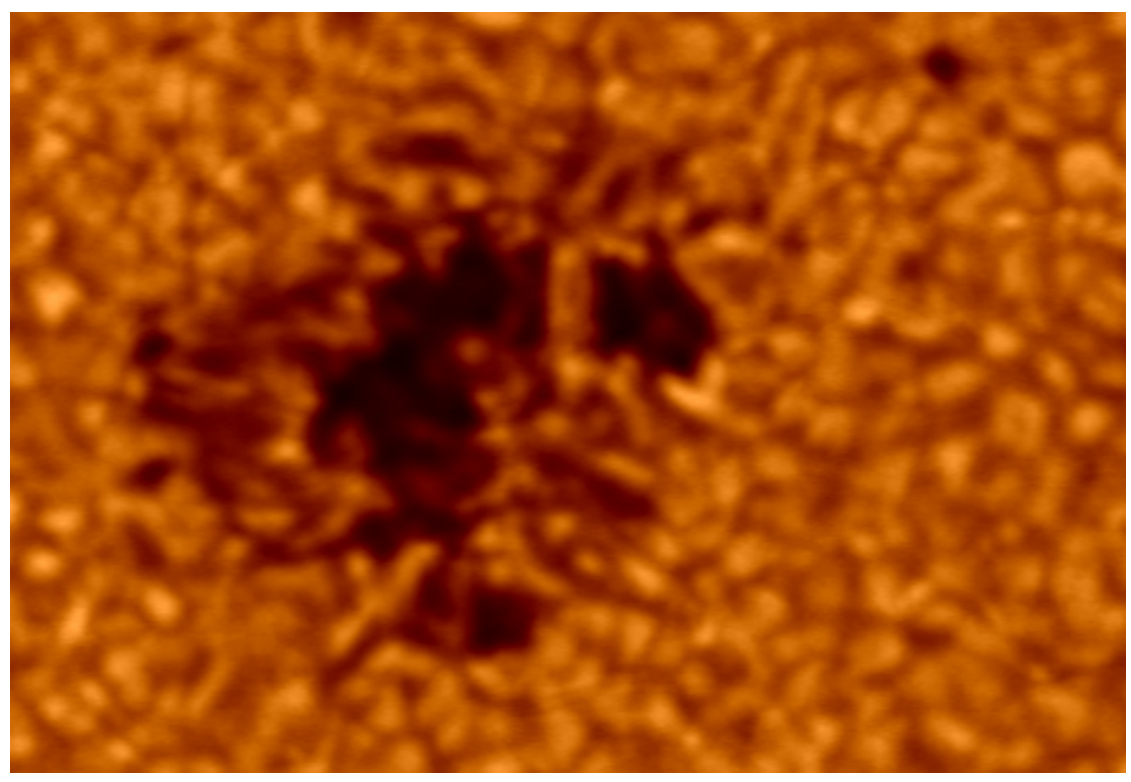

Fig. 2 (c) Iteratively reconstructed SAA image, where an SAA reconstructed image, instead of the best-shot image, is used for the reference frame in the next iterative step. 